\title{
On Z-Projective Change of Kropina Spaces
}

\author{
Salim Ceyhan* Gülçin Çivi
}

(Communicated by Levent KULA)

\begin{abstract}
In this paper, we consider the projective change $\sigma: F \rightarrow \widetilde{F}$ of metrics of the Kropina space $F_{n}$ and the Finsler space $\widetilde{F}_{n}$, respectively. It is known that The Douglas and the Weyl Curvature tensors remain invariant under the projective change of the Finsler metrics. Moreover, $h$-curvature tensor in the Berwald connection is invariant under the a special projective change named as $Z$-projective change. M. Fukui and T. Yamada studied in the projective change between two Finsler spaces. Then, B.D. Kim and H.Y. Park proved that if a symmetric space remains to be symmetric one under the $Z$-projective change then the space is of zero curvature.

In present paper, we first investigated in the quantities which are invariant under the $Z$ - projective change between two Finsler spaces. Then, we obtained the necessary and sufficient conditions for a projective change $\sigma: F \rightarrow \widetilde{F}$ between a Kropina space $F_{n}(n>2)$ and a Finsler space $\widetilde{F}_{n}(n>2)$ to be a $Z$-projective change.
\end{abstract}

Keywords: Finsler space; R-curvature tensor; Riemannian curvature; Z-projective change.

AMS Subject Classification (2010): Primary: 53B40 ; Secondary: 53A20.

\section{Introduction}

A Minkowski norm on a vector space $V$ is a nonnegative function $F: V \rightarrow[0, \infty)$ with following properties:

1. $F(y) \geq 0$ for any $y \in V$ and $F(y)=0 \Leftrightarrow y=0$,

2. $F(\lambda y)=\lambda F(y)$, for any $\lambda>0$ and any $y \in V$, i.e. $F$ is positively $y$-homogeneous of degree one,

3. $F(y)$ is on $V \backslash\{0\}$ such that the matrix

$$
g_{i j}(y)=\frac{1}{2}\left[F^{2}\right]_{y^{i} y^{j}}(y)
$$

is positive definite. On an $n$-dimesional manifold $M$, a Finsler metric $F$ is a $C^{\infty}$ function on $T M_{0}=T M \backslash\{0\}$ such that $F_{x}=F_{T_{x} M}$ is a Minkowski norm on $T_{x} M$ for any $x \in M$.

Let $F$ be a Finsler metric on $M$. The pair $(M, F)$ is called a Finsler space. In a Finsler space, the metric tensor is given by

$$
g_{i j}(x, y)=\frac{1}{2} \frac{\partial^{2} F^{2}}{\partial y^{i} \partial y^{j}}(x, y),
$$

where $x=x^{i}$ denotes the coordinates of $p \in M$ and $(x, y)=\left(x^{i}, y^{i}\right)$ denotes the local coordinates of $y \in$ $T_{p} M[8,7]$.

In local coordinates, the geodesics of $F=F(x, y)$ Finsler metrics are characterized by a system of equations:

$$
\frac{d^{2} x^{i}}{d t^{2}}+2 G^{i}\left(x, \frac{d x}{d t}\right)=0,
$$

Received : 03-09-2015, Accepted : 18-02-2016

* Corresponding author

This article is the written version of author's plenary talk delivered on September 03-August 31, 2015 at 4th International Eurasian Conference on Mathematical Sciences and Applications (IECMSA-2015) in Athens, Greece. 
where $G^{i}$ is called the geodesics spray coefficients denoted by

$$
G^{i}:=\frac{1}{4} g^{i l}\left\{\left[F^{2}\right]_{x^{k} y^{l}} y^{k}-\left[F^{2}\right]_{x^{l}}\right\}
$$

and these coefficients are positive functions with $y$-homogeneous of the second degree. The functions $G^{i}$ gives a global vector field called spray on $T M_{0}[6]$.

In Finsler geometry, Finsler metrics are separated into several classes according to their geometric properties. In this work, we concerned with a Finsler space with Kropina metric.

Let $\phi=\phi(s)$ be a $C^{\infty}$ positive function on an open interval $\left(-b_{0}, b_{0}\right)$ satisfying

$$
\phi(s)-s \phi^{\prime}(s)+\left(b^{2}-s^{2}\right) \phi^{\prime \prime}(s)>0,|s| \leq b<b_{0},
$$

where $b^{2}=a^{i j}(x) b_{i} b_{j}=b_{i} b^{i}, b_{i}=b_{i}(x), b=\left\|\beta_{x}\right\|_{\alpha}=\sqrt{a^{i j}(x) b_{i} b_{j}}<b_{0}$ and $\alpha=\sqrt{a_{i j}(x) y^{i} y^{j}}, \beta=b_{i}(x) y^{i}$ respectively, a Riemannian metric and differantial 1 -form[4, 7].

Kropina metric is a special class of $(\alpha, \beta)$-metrics having the form

$$
F=\frac{\alpha^{2}}{\beta} \text {. }
$$

\subsection{Definition of Projective Change}

Two Finsler metrics $F$ and $\widetilde{F}$ on a manifold $M$ are said to be (pointwise) projectively related if they have the same geodesics as point sets. $\sigma: F \rightarrow \widetilde{F}$ metric change between two Finsler metrics $F$ and $\widetilde{F}$ on a manifold $M$ is called projective change. Projective change has been characterized using spray coefficients.

Let $\widetilde{F}_{n}$ and $F_{n}$ be two Finsler spaces. The Finsler metrics $\widetilde{F}$ and $F$ are projectively related to each other if and only if there exists a scalar function $P$ on $T M_{0}$. In this case, we can write

$$
G_{F}^{i}=G_{F}^{i}+P(x, y) y^{i},
$$

where $P$ is homogeneous of degree one in $y$ and $G_{F}^{i}$ and $G_{F}^{i}$ are the spray coefficients of $F$ and $\widetilde{F}$, respectively[5].

\subsection{Relation of Spray Coefficients}

The relation between the spray coefficients of $(\alpha, \beta)$-metric, $G_{F}^{i}$ and the Riemannian metric $\alpha, G_{\alpha}^{i}=\frac{1}{2} \Gamma_{j k}^{i} y^{j} y^{k}$ are given by

$$
\begin{aligned}
G_{F}^{i}= & G_{\alpha}^{i}+\frac{\alpha \phi^{\prime}}{\phi-s \phi^{\prime}} s_{0}^{i}+\frac{\phi \phi^{\prime}-s\left(\phi \phi^{\prime \prime}+\phi^{\prime} \phi^{\prime}\right)}{2 \phi\left[\left(\phi-s \phi^{\prime}\right)+\left(b^{2}-s^{2}\right) \phi^{\prime \prime}\right]}\left(\frac{-2 \alpha \phi^{\prime}}{\phi-s \phi^{\prime}} s_{0}+r_{00}\right) \times \\
& \left(\frac{y^{i}}{\alpha}+\frac{\phi \phi^{\prime \prime}}{\phi \phi^{\prime}-s\left(\phi \phi^{\prime \prime}+\phi^{\prime} \phi^{\prime}\right)} b^{i}\right)
\end{aligned}
$$

where $\Gamma_{j k}^{i}=\Gamma_{j k}^{i}(x)$ are the second kind of Christoffel symbols, and

$$
\begin{aligned}
r_{i j} & =\frac{1}{2}\left(b_{i ; j}+b_{j ; i}\right), s_{i j}=\frac{1}{2}\left(b_{i ; j}-b_{j ; i}\right), s_{j}^{i}=a^{i r} s_{r j}, s_{0}^{i}=s_{j}^{i} y^{j}, s_{j}=s_{i j} b^{i}, \\
s_{0} & =s_{i} y^{i}, r_{00}=r_{i j} y^{i} y^{j}, b^{2}=a^{i j} b_{i} b_{j}
\end{aligned}
$$

and ";" denotes the covariant derivative with respect to Levi-Civita connection of the Riemannian $\alpha$-metric[1].

By using this relation we have the following lemma.

Lemma 1.1. The spray coefficients $G_{F}^{i}$ of the Kropina metric $F$ and the spray coefficients $G_{\alpha}^{i}$ of the Riemannian metric $\alpha$ are related by

$$
G_{F}^{i}=G_{\alpha}^{i}-\frac{\alpha^{2}}{2 \beta} s_{0}^{i}+\frac{\alpha^{2} s_{0}+\beta r_{00}}{2 \beta b^{2}} b^{i}-\frac{\alpha^{2} s_{0}+\beta r_{00}}{\alpha^{2} b^{2}} y^{i} .
$$

Proof. Since $\phi(s)=\frac{1}{s}$, for a Kropina metric, substituting $\phi(s)$ in (1.1), the relation between the spray coefficients of Kropina and Riemannian metric is obtained as (1.2). 
By substituting

$$
\begin{aligned}
G_{F}^{i} & =G_{\alpha}^{i}-\frac{\alpha^{2}}{2 \beta} s_{0}^{i}+\frac{\alpha^{2} s_{0}+\beta r_{00}}{2 b^{2} \beta} b^{i} \\
& =G_{\alpha}^{i}+\Omega^{i}, \quad\left(\Omega^{i}=-\frac{\alpha^{2}}{2 \beta} s_{0}^{i}+\frac{\alpha^{2} s_{0}+\beta r_{00}}{2 b^{2} \beta} b^{i}\right) .
\end{aligned}
$$

In (1.2), we obtain,

$$
G_{F}^{i}=G_{F}^{i}+P y^{i}
$$

which characterizes the spray $G_{F}^{i}$ is projectively equivalent to $G_{F}^{i}$, where projective factor $P$ is

$$
P(x, y)=\frac{1}{b^{2}}\left(s_{0}+\frac{\beta}{\alpha^{2}} r_{00}\right) .
$$

\section{Z-Projective Finsler Spaces}

The projective change, whose projective factor $P(x, y)$, satisfying the following conditions

$$
P_{\mid i}-P P_{i}=0, \quad\left(P_{i}=\frac{\partial P}{\partial y^{i}}, P_{\mid i}=\frac{\partial P}{\partial x^{i}}-G_{F i}^{r} \frac{\partial P}{\partial y^{r}}\right) .
$$

is called $Z$-projective change, where the symbol "|" denotes the horizontal covariant derivative with respect to the Berwald connection $B \Gamma\left(G_{F j k}^{i}, G_{F j}^{i}, 0\right)$ of $F$ Finsler metric[2]. The connection coefficients of $G_{F j}^{i}$ and $G_{F j k}^{i}$ of $B \Gamma$ can be derived from the functions $G^{i}$, namely $G_{F j}^{i}=\frac{\partial G_{F}^{i}}{\partial y^{j}}$ and $G_{\digamma F k}^{i}=\frac{\partial G_{F j}^{i}}{\partial y^{k}}$.

\subsection{The Quantities Which Are Invariant Under The Z-Projective Change}

The Douglas and the Weyl curvature tensors of $F_{n}$ Finsler space are defined by

$$
D_{i j k}^{h}=G_{F i j k}^{h}-\frac{1}{n+1}\left(y^{h} \frac{\partial G_{F i j}}{\partial y^{k}}+\delta_{i}^{h} G_{F j k}+\delta_{k}^{h} G_{F i j}+\delta_{j}^{h} G_{F k i}\right)
$$

and

$$
W_{k}^{i}=R_{k}^{i}-R \delta_{k}^{i}-\frac{1}{n+1} \frac{\partial}{\partial y^{m}}\left(R_{k}^{m}-R \delta_{k}^{m}\right) y^{i}
$$

respectively, where

$$
G_{F i j k}^{h}=\frac{\partial G_{i j}^{h}}{\partial y^{k}}, G_{F i j}=G_{F i j r}^{r}
$$

and

$$
R=\frac{1}{n-1} R_{m}^{m}
$$

$R_{m}^{m}$ is the Ricci curvature(or Ricci scalar)[1].

It is known that The Douglas and the Weyl curvature tensors remain invariant under the projective change of two Finsler metrics.

In this work, we investigate the other quantities, which are invariant under the $Z$-projective change between two Finsler spaces except the Douglas and the Weyl curvature tensors.

Theorem 2.1. Riemannian curvature tensor of a Finsler metric is invariant under the projective change if and only if $P$, projective factor of change is satisfied the following equations:

$$
P_{\mid k}-P_{k \mid 0}=0, \quad\left(P_{k \mid 0}=P_{k \mid l} y^{l}\right)
$$


Proof. In $F_{n}$ Finsler space with $B \Gamma\left(G_{F j k}^{i}, G_{F j}^{i}, 0\right)$ Berwald connection, $R_{F k}^{i}$ coefficients of Riemann tensor are defined by

$$
R_{F}^{i}=2 \frac{\partial G_{F}^{i}}{\partial x^{k}}-y^{j} \frac{\partial^{2} G_{F}^{i}}{\partial x^{j} \partial y^{k}}+2 G_{F}^{j} \frac{\partial^{2} G_{F}^{i}}{\partial y^{j} \partial y^{k}}-\frac{\partial G_{F}^{i}}{\partial y^{j}} \frac{\partial G_{F}^{j}}{\partial y^{k}}
$$

[1].

Under the condition (2.1), the equations (2.3) and (1.3) lead to

$$
R_{\tilde{F} k}^{i}=R_{F k}^{i}+\left(2 P_{\mid k}-P_{k \mid 0}-P P_{k}\right) y^{i}-\left(P_{\mid 0}-P^{2}\right) \delta_{k}^{i},
$$

where $P_{\mid k}=\frac{\partial P}{\partial x^{k}}-G_{F k}^{r} \frac{\partial P}{\partial y^{r}}, P_{\mid 0}=P_{\mid k} y^{k}$.

Assume that the Riemannian curvature tensor is an invariant than we have

$$
R_{\widetilde{F} k}^{i}=R_{F k}^{i} .
$$

Thus (2.4) reduces to

$$
\left(P P_{k}-P_{k \mid 0}\right) y^{i}-\left(P_{\mid 0}-P^{2}\right) \delta_{k}^{i}=0
$$

where the second term in (2.5) is

$$
P_{\mid 0}-P^{2}=\left(P_{\mid k}-P P_{k}\right) y^{k}=0
$$

and substituting (2.1) into (2.5) we obtain

$$
P_{\mid k}-P_{k \mid 0}=0
$$

Conversely (2.1) and (2.2) in (2.4) we find

$$
\begin{aligned}
R_{\tilde{F} k}^{i} & =R_{F k}^{i}+\left(2 P_{\mid k}-P_{k \mid 0}-P P_{k}\right) y^{i}-\left(P_{\mid 0}-P^{2}\right) \delta_{k}^{i} \\
& =R_{F k}^{i}+\left(P_{\mid k}-P_{k \mid 0}\right) y^{i} \\
& =R_{F k}^{i} .
\end{aligned}
$$

which completes the proof.

Theorem 2.2. $R_{F l j k}^{i}, R$-curvature tensor of a $F_{n}$ Finsler space is invariant under the $Z$-projective change.

Proof. Let $F_{n}$ be a Finsler space with $B \Gamma\left(G_{F j k}^{i}, G_{F j}^{i}, 0\right)$ connection. Differentiating $R_{F j k}^{i}$ defined by

$$
R_{F j k}^{i}=\partial_{k} G_{F j}^{i}-\partial_{j} G_{F k}^{i}+G_{F k r}^{i} G_{F j}^{r}-G_{F j r}^{i} G_{F k}^{r},
$$

with respect to $y^{l}$ give the $R$-curvature tensor[3].

$$
\begin{aligned}
R_{F l j k}^{i} & =\dot{\partial}_{l} R_{F j k}^{i} \\
& =\partial_{k} G_{F j l}^{i}-\partial_{j} G_{F k l}^{i}-G_{F k}^{r} \dot{\partial}_{l} G_{F j r}^{i}+G_{F j}^{r} \dot{\partial}_{l} G_{F k r}^{i}+G_{F k r}^{i} G_{F j l}^{r}-G_{F j r}^{i} G_{F k l}^{r},
\end{aligned}
$$

where $\partial_{k}=\frac{\partial}{\partial x^{k}}, \dot{\partial}_{k}=\frac{\partial}{\partial y^{k}}$.

It is known that, for a Berwald space the following conditions

$$
\dot{\partial}_{l} G_{F k r}^{i}=\dot{\partial}_{l} G_{F j r}^{i}=0
$$

holds. Then (2.6) reduces to

$$
R_{F l j k}^{i}=\dot{\partial}_{l} R_{F j k}^{i}=\partial_{k} G_{F j l}^{i}-\partial_{j} G_{F k l}^{i}+G_{F k r}^{i} G_{F j l}^{r}-G_{F j r}^{i} G_{F k l}^{r} .
$$

Under any projective change between two spaces with $F$ and $\widetilde{F}$ Finsler metrics, using the relation $G_{\widetilde{F}}^{i}=$ $G_{F}^{i}+P y^{i}$ of the spray coefficents is obtained as

$$
\begin{aligned}
G_{\tilde{F} j}^{i} & =G_{\tilde{F} j}^{i}+y^{i} P_{j}+P \delta_{j}^{i} \\
G_{\tilde{F} j k}^{i} & =G_{\tilde{F} j k}^{i}+y^{i} P_{j k}+P_{j} \delta_{k}^{i}+P_{k} \delta_{j}^{i} \\
\dot{\partial}_{l} G_{\tilde{F} k r}^{i} & =G_{\tilde{F} k r l}^{i}=G_{\tilde{F} k r l}^{i}+y^{i} P_{k r l}+\delta_{k}^{i} P_{r l}+\delta_{r}^{i} P_{k l}+\delta_{k}^{l} P_{k r},
\end{aligned}
$$


where $P_{i}=\dot{\partial}_{i} P$ ve $P_{i j}=\dot{\partial}_{j} P_{i}$. Using (2.6) and (2.8), $R$-curvature tensor of $\widetilde{F}_{n}$ is written as

$$
\begin{aligned}
R_{\tilde{F}}^{i} l j k & =\partial_{k} G_{\tilde{F} j l}^{i}-\partial_{j} G_{\tilde{F} k l}^{i}-G_{\tilde{F} k}^{r} \dot{\partial}_{l} G_{\tilde{F} j j}^{i}+G_{\tilde{F} j}^{r} \dot{\partial}_{l} G_{\tilde{F} k r}^{i}+G_{\tilde{F} k r}^{i} G_{\tilde{F} j l}^{r}-G_{\tilde{F} j r}^{i} G_{\tilde{F}}^{r} k l \\
& =R_{F l j k}^{i}+y^{i} \dot{\partial}_{l} \Sigma_{j k}+\delta_{l}^{i} \Sigma_{j k}+\delta_{j}^{i} \dot{\partial}_{l} \Sigma_{k}-\delta_{k}^{i} \dot{\partial}_{l} \Sigma_{j},
\end{aligned}
$$

where $\Sigma_{i}=P_{\mid i}-P P_{i}$ and $\Sigma_{j k}=P_{j \mid k}-P_{k \mid j}=\dot{\partial}_{j} \Sigma_{k}-\dot{\partial}_{k} \Sigma_{j}$. Under $Z$-projective condition, setting $\Sigma_{i}=0$ and $\Sigma_{j k}=0$ in (2.9) yields to

$$
R_{\widetilde{F}}^{i} l j k=R_{F l j k}^{i},
$$

which completes the proof.

\subsection{Z-Projective Kropina Spaces}

A projective change of a Finsler space of zero curvature is also a Finsler space of zero curvature if and only if the projective factor $P$ satisfies the equation (2.1). In other words, projective change

Theorem 2.3. The necessary and sufficient conditions for which the projective change between a Kropina space $F_{n}(n>2)$ and a Finsler space $\widetilde{F}_{n}(n>2)$ is a $Z$-projective change are

$$
\begin{aligned}
& r_{00}=c(x) \alpha^{2}, \\
& b^{2} s_{0}^{i} s_{i}+\beta s^{i} s_{i}=0, \\
& b^{2} s_{j \mid i}-c b_{i} s_{j}-s_{i} s_{j}+b^{2} c s_{j i}-s^{r} s_{r} a_{i j}=0,
\end{aligned}
$$

where $c=c(x)$ is a differentiable fonction satisfying the conditions

$$
b^{2} c_{\mid i}-c^{2} b_{i}-c s_{i}=0 .
$$

Proof. According to Lemma 1.1, the relation between spray coefficients of Kropina and Riemann space from is

$$
G_{F}^{i}=G_{\alpha}^{i}-P y^{i}+\Omega^{i} .
$$

or putting

$$
\begin{aligned}
G_{\widetilde{F}}^{i} & =G_{\alpha}^{i}+\Omega^{i} \\
& =G_{\alpha}^{i}-\frac{\alpha^{2}}{2 \beta} s_{0}^{i}+\frac{\alpha^{2} s_{0}+\beta r_{00}}{2 \beta b^{2}} b^{i}
\end{aligned}
$$

we get

$$
G_{\widetilde{F}}^{i}=G_{F}^{i}+P y^{i}
$$

which defines a projective change between $F$ Kropina metric and $\widetilde{F}$ Finsler metric. Let $\sigma: F \rightarrow \widetilde{F}$ be a projective change. If $\sigma$ is a $Z$-projective change than the projective factor $P(x, y)$ satisfies the conditions

$$
P_{\mid i}-P P_{i}=0,
$$

where

$$
P=\frac{1}{b^{2}}\left(s_{0}+\beta \frac{r_{00}}{\alpha^{2}}\right) .
$$

By taking the horizontal covariant derivative of and the partial derivative of $P$ given by

$$
P_{\mid i}=\frac{\partial P}{\partial x^{i}}-G_{F i}^{r} \frac{\partial P}{\partial y^{r}}
$$

and

$$
P_{i}=\frac{\partial P}{\partial y^{i}},
$$

respectively in (2.13) we obtain

$$
\begin{aligned}
& 4 r_{00} \beta^{4}\left\{2 \alpha^{2} r_{i 0}-y_{i} r_{00}\right\}+2 \beta^{3} \alpha^{2}\left\{\left[-2 r_{00} r_{i}+b^{2} r_{00 \mid i}+2 r_{i 0}\left(s_{0}-r_{0}\right)\right] \alpha^{2}+b_{i} r_{00}^{2}\right\} \\
& +2 \beta^{2} \alpha^{4}\left\{\left[b^{2} s_{0 \mid i}-s_{i}\left(r_{0}+s_{0}\right)+b^{2} s_{i}^{r} r_{r 0}-2 s_{0} r_{i}\right] \alpha^{2}+2 y_{i}\left(b^{2} s_{0}^{r} r_{r 0}-s_{0} r_{0}\right)\right\} \\
& +\beta \alpha^{6}\left\{\alpha^{2} b^{2} s_{i}^{r} s_{r}-2 b^{2} b_{i} s_{0}^{r} r_{r 0}+2 b^{2} y_{i} s_{0}^{r} s_{r}+2 b_{i} s_{0} r_{0}\right\}-\alpha^{8} b^{2} b_{i} s_{0}^{r} s_{r}=0
\end{aligned}
$$


which denote polynomial equations with 9th-degree. These equations are implicitly in the following form:

$$
A_{5} \beta^{4}+A_{4} \beta^{3} \alpha^{2}+A_{3} \beta^{2} \alpha^{4}+A_{2} \beta \alpha^{6}+A_{1} \alpha^{8}=0,
$$

where the coefficients of $A_{i}, i=1, \cdots, 5$ are polynomials in $y$ and the subscripts denote the degree of homogenity with respect to $y$. It is clear that coefficients are in the form

$$
\begin{aligned}
& A_{5}=4 r_{00}\left(2 \alpha^{2} r_{i 0}-y_{i} r_{00}\right), \\
& A_{4}=2\left[\left(-2 r_{00} r_{i}+b^{2} r_{00 \mid i}+2 r_{i 0}\left(s_{0}-r_{0}\right)\right) \alpha^{2}+b_{i} r_{00}^{2}\right], \\
& A_{3}=2\left[\left(b^{2} s_{0 \mid i}-s_{i}\left(r_{0}+s_{0}\right)+b^{2} s_{i}^{r} r_{r 0}-2 s_{0} r_{i}\right) \alpha^{2}+2 y_{i}\left(b^{2} s_{0}^{r} r_{r 0}-s_{0} r_{0}\right)\right], \\
& A_{2}=\alpha^{2} b^{2} s_{i}^{r} s_{r}-2 b^{2} b_{i} s_{0}^{r} r_{r 0}+2 b^{2} y_{i} s_{0}^{r} s_{r}+2 b_{i} s_{0} r_{0}, \\
& A_{1}=-b^{2} b_{i} s_{0}^{r} s_{r} .
\end{aligned}
$$

Since the term $A_{1}=-b^{2} b_{i} s_{0}^{r} s_{r}$ does not contain $\beta$, there must be a scalar function $f_{i}(x)$ such that

$$
b^{2} b_{i} s_{0}^{r} s_{r}=\beta f_{i}(x) .
$$

Differentiating both sides of (2.16) with respect to $y^{j}$ and considering

$$
f_{i}(x)=-b_{i} s^{r} s_{r}
$$

the equation (2.16) can be written as

$$
b_{i}\left(b^{2} s_{0}^{r} s_{r}+\beta s^{r} s_{r}\right)=0
$$

or

$$
b^{2} s_{0}^{r} s_{r}+\beta s^{r} s_{r}=0 .
$$

On the other hand substituting (2.17) in (2.15) and paying attention to the terms of $A_{2}+A_{1}=-2 b^{2} b_{i} s_{0}^{r} r_{r 0}+$ $2 b_{i} s_{0} r_{0}$, which seemingly does not contain $\beta$, it is seen that needed to stasfying,

$$
b_{i}\left(s_{0} r_{0}-b^{2} s_{0}^{r} r_{r 0}\right)=\beta u_{i 1},
$$

where $u_{i 1}=u_{i j}(x) y^{j}$. Differentiating both sides of (2.18) with respect to $y^{j}$ and $y^{k}$ and transvecting the equations obtained by differentiation by $b^{j}$ and $b^{k}$ give

$$
b_{i} s_{j} r_{k} b^{k}-b_{i} b^{2}\left(-r_{j r} s^{r}+r_{r} s_{j}^{r}\right)=b_{j} u_{i k} b^{k}+b^{2} u_{i j}
$$

and

$$
b_{i} s^{r} r_{r}=u_{i r} b^{r},
$$

respectively.

Substituting (2.20) into (2.19), we obtain

$$
\begin{aligned}
u_{i j}(x) & =\frac{b_{i}}{b^{2}}\left[s_{j} r_{r} b^{r}-b_{j} s^{r} r_{r}+b^{2}\left(r_{j r} s^{r}-s_{j}^{r} r_{r}\right)\right], \\
u_{i 1} & =u_{i j}(x) y^{j}=\frac{b_{i}}{b^{2}}\left[s_{0} r_{r} b^{r}-\beta s^{r} r_{r}+b^{2}\left(r_{0 r} s^{r}-s_{0}^{r} r_{r}\right)\right] .
\end{aligned}
$$

if (2.21) is multiplied by $b^{i}$, we have

$$
u_{i 1} b^{i}=s_{0} r_{r} b^{r}-\beta s^{r} r_{r}+b^{2}\left(r_{0 r} s^{r}-s_{0}^{r} r_{r}\right) .
$$

Then, substituting $u_{i 1}$ into (2.15), we obtain

$$
\begin{aligned}
& \alpha^{6}\left\{b^{2} s_{0 \mid i}-s_{i}\left(r_{0}+s_{0}\right)+b^{2} s_{i}^{r} r_{r 0}-2 s_{0} r_{i}-s^{r} s_{r} y_{i}+\frac{b_{i}}{b^{2}}\left[s_{0} r_{r} b^{r}-\beta s^{r} r_{r}\right.\right. \\
& \left.\left.+b^{2}\left(r_{0 r} s^{r}-s_{0}^{r} r_{r}\right)\right]\right\}+\alpha^{4} \beta\left\{-2 r_{00} r_{i}+b^{2} r_{00 \mid i}+2 r_{i 0}\left(s_{0}-r_{0}\right)\right. \\
& \left.-\frac{2}{b^{2}} y_{i}\left[s_{0} r_{r} b^{r}-\beta s^{r} r_{r}+b^{2}\left(r_{0 r} s^{r}-s_{0}^{r} r_{r}\right)\right]\right\}+\alpha^{2} \beta r_{00}\left(4 \beta r_{i 0}+r_{00} b_{i}\right) \\
& -2 \beta^{2} r_{00}^{2} y_{i}=0
\end{aligned}
$$


which do not contain $\beta$. Then we can write

$$
b^{2} s_{0 \mid i}-s_{i}\left(r_{0}+s_{0}\right)+b^{2} s_{i}^{r} r_{r 0}-2 s_{0} r_{i}-s^{r} s_{r} y_{i}+\frac{b_{i}}{b^{2}}\left[s_{0} r_{r} b^{r}+b^{2}\left(r_{0 r} s^{r}-s_{0}^{r} r_{r}\right)\right]=\beta g_{i}(x) .
$$

Differentiating (2.23) by $y^{j}$ and resulting equation transvecting $b^{j}$, we find $g_{i}(x)$ fonction as

$$
g_{i}(x)=-s^{r}\left(s_{r i}+r_{r i}\right)+s_{i}^{r} r_{r}-\frac{1}{b^{2}} s_{i} r_{r} b^{r}+\frac{1}{b^{2}}\left(2 s^{r} r_{r}-s^{r} s_{r}\right) b_{i} .
$$

Consequently, multiplying (2.22) by $b^{i}$ and paying attention to be $u_{i 1} b^{i}=\frac{b^{2}}{\beta}\left(s_{0} r_{0}-b^{2} s_{0}^{r} r_{r 0}\right), g_{i}(x) b^{i}=0$, we find

$$
\begin{aligned}
& \alpha^{2}\left\{\alpha^{4}\left(-s^{i} r_{i}\right)+\alpha^{2}\left(b^{2} b^{i} r_{00 \mid i}-2 r_{00} r_{i} b^{i}-2 r_{0}^{2}+2 b^{2} s_{0}^{i} r_{i 0}\right)+b^{2} r_{00}^{2}\right\} \\
& =2 \beta r_{00}\left(\beta r_{00}-2 \alpha^{2} r_{0}\right) .
\end{aligned}
$$

For $n>2, \alpha^{2} \not \equiv 0(\bmod \beta)$ and $b^{2} \neq 0$, term on the right side of (2.25) must be a multiple of $\alpha^{2}$. According to this, for the realizing of (2.25) we get

$$
r_{00}=c \alpha^{2}
$$

or

$$
r_{00} \beta-2 r_{0} \alpha^{2}=\alpha^{2} v_{1},
$$

where $v_{1}=v_{i}(x) y^{i}$ and $c=c(x)$ are scalar functions of $x$. It is easily seen that (2.26) and (2.27) are equivalent to each other. On the other hand, substituting the conditions $r_{00}=c \alpha^{2}$ and $b^{2} s_{0}^{r} s_{r}+\beta s^{r} s_{r}=0$ into (2.14) after necessarly calculations, we find

$$
b^{2} s_{j \mid i}-c b_{i} s_{j}-s_{i} s_{j}+b^{2} c s_{j i}-s^{r} s_{r} a_{i j}=0
$$

and the scalar function $c(x)$ satisfying

$$
b^{2} c_{\mid i}-c^{2} b_{i}-c s_{i}=0 .
$$

Taking account of this conditions, we find the necessary conditions for a projective change to be a $Z$-projective change as

and

$$
b^{2} s_{0}^{r} s_{r}+\beta s^{r} s_{r}=0, r_{00}=c \alpha^{2}
$$

$$
b^{2} s_{j \mid i}-c b_{i} s_{j}-s_{i} s_{j}+b^{2} c s_{j i}-s^{r} s_{r} a_{i j}=0 .
$$

Conversely, substituting (2.10) conditions into $P_{\mid i}-P P_{i}$, we find

$$
P_{\mid i}-P P_{i}=0,
$$

which completes the proof.

Corollary 2.1. In theorem 2.3, the necessary and sufficient conditions for which the projective change between $F$ Kropina metric with weak Berwald space and a $\widetilde{F}_{n}$ Finsler metric is a $Z$-projective change are

$$
b^{2} s_{0}^{r} s_{r}+\beta s^{r} s_{r}=0
$$

and

$$
b^{2} s_{j \mid i}-c b_{i} s_{j}-s_{i} s_{j}+b^{2} c s_{j i}-s^{r} s_{r} a_{i j}=0,
$$

where $c=c(x)$ is a scalar function satisfying the conditions

$$
b^{2} c_{\mid i}-c^{2} b_{i}-c s_{i}=0 .
$$

\section{References}

[1] Chern, S.S. and Shen, Z., Riemann-Finsler Geometry, World Scientific, Singapore, New Jersey, London, Hong Kong, 2005.

[2] Fukui, M. and Yamada, T., On projective mapping in Finsler geometry, Tensor, N.S., 35 (2000), $216-222$.

[3] Kim, B-D. and Park, Ha-Y., On special Finsler spaces with common geodesics, Korean Math. Soc., 15 (2000), $331-338$.

[4] Matsumoto, M., Finsler spaces with $(\alpha, \beta)$-metric of Douglas type, Tensor, N.S., 60 (1998), 123-134.

[5] Nagaraja, H.G., On projective changes of Finsler metrics, Tensor, N.S., 65 (2004), 181-188.

[6] Peyghan, E. and Tayebi, A., Generalized Berwald Metrics, Turkish Journal Math., 35 (2011), 1-10.

[7] Shen, Z., Differential geometry of spray and Finsler spaces, Kluwer Academic Publishers, Dordrecht, 2001.

[8] Shen, Z., Lectures on Finsler geometry, World Scientific, Singapore, New Jersey, London, Hong Kong, 2001. 


\section{Affiliations}

SALIM CEYHAN

AdDrESS: Bilecik Şeyh Edebali University, Dept. of Computer Engineering, 11210, BİLECİK-TURKEY. E-MAIL: salim.ceyhan@bilecik.edu.tr

GÜLÇIN ÇIVI

ADDRESS: Istanbul Technical University, Dept. of Mathematics, 34469, ISTANBUL-TURKEY.

E-MAIL: civi@itu.edu.tr 